\title{
Physical Activity, Central Adiposity, and Functional Limitations in Community-Dwelling Older Adults
}

\author{
Cassandra M. Germain, $\mathrm{PhD}^{1}$; Elizabeth Vasquez, $\mathrm{DrPH}^{2}$; John A. Batsis, $\mathrm{MD}^{3}$
}

\begin{abstract}
Background and Purpose: Obesity and physical inactivity are independently associated with declines in physical and functional limitations in older adults. The current study examines the impact of physical activity on odds of physical and functional limitations in older adults with central and general obesity.
\end{abstract}

Methods: Data from 6279 community-dwelling adults aged 60 years or more from the Health and Retirement Study 2006 and 2008 waves were used to calculate prevalence and odds of physical and functional limitation among obese older adults with high waist circumference (waist circumference $\geq 88 \mathrm{~cm}$ in females and $\geq 102 \mathrm{~cm}$ in males) who were physically active versus inactive (engaging in moderate/vigorous activity less than once per week). Logistic regression models were adjusted for age, sex, race/ethnicity, education, smoking status, body mass index, and number of comorbidities.

Results: Physical activity was associated with lower odds of physical and functional limitations among older adults with high waist circumference (odds ratio [OR], 0.59; confidence interval [Cl], 0.52-0.68, for physical limitations; OR, 0.52; Cl,

\section{${ }^{1}$ Department of Psychiatry and Behavioral Sciences, Duke University Medical Center, Durham, North Carolina. \\ ${ }^{2}$ Department of Epidemiology and Biostatistics, School of Public Health, University at Albany (SUNY), One University Place, Albany, Rensselaer, New York. \\ ${ }^{3}$ Department of Medicine, Geisel School of Medicine at Dartmouth, Dartmouth-Hitchcock Medical Center, Lebanon, New Hampshire.}

Dr Germain is supported in part by NHLBI IRS Diversity Supplement \#3R01HL109219-02S1. Dr Batsis is partially supported by UB4HP19206-01-00 as a Medical Educator from the Health Resources and Services Administration, the Dartmouth Centers for Health and Aging, and has received support from the Department of Medicine, DartmouthHitchcock Medical Center. He has served as a consultant to the legal firm Dinse, Knapp \& McAndrew LLC for a clinical case review in 2012. This project was initiated and analyzed solely by the investigators.

The authors declare no conflicts of interest.

Address correspondence to: Cassandra M. Germain, PhD, Duke University Medical Center, Department of Psychiatry and Behavioral Sciences, Box 3119, Durham, NC 27710 (cassandra.germain@duke.edu).

Marybeth Brown was the Decision Editor.

DOI: 10.1519/JPT.0000000000000051
0.44-0.62, for activities of daily living; and $\mathrm{OR}, 0.44 ; \mathrm{Cl}, 0.39$ 0.50 , for instrumental activities of daily living).

Conclusions: Physical activity is associated with significantly lower odds of physical and functional limitations in obese older adults regardless of how obesity is classified. Additional research is needed to determine whether physical activity moderates long-term physical and functional limitations.

Key Words: functional limitations, obesity, older adults, physical activity, prevalence, waist circumference

( J Geriatr Phys Ther 2015;38:1-7.)

\section{INTRODUCTION}

Obesity is associated with a variety of poor health outcomes including cardiovascular disease, increased prevalence of medical comorbidities, and functional disability. ${ }^{1-3}$ Approximately $35.5 \%$ of adults in the United States are currently obese. ${ }^{4}$ This represents an almost 3 -fold increase from 1960 to present and continues to rise, even among older adults. ${ }^{5}$ Among those aged 60 years and more, the combined prevalence of overweight and obesity, as assessed by body mass index (BMI), is estimated at more than $70 \% .{ }^{6}$ Concurrently, the prevalence of abdominal adiposity is also rising in the United States across all age groups. ${ }^{7}$ This growing trend in central adiposity represents an important public health concern given that excess abdominal fat is independently associated with disability among older adults. ${ }^{8}$ Although general adiposity (as measured by BMI) remains the most widely used marker of obesity in clinical settings and epidemiological studies, accumulating evidence suggests that abdominal adiposity may be a more sensitive predictor of obesity-related health problems and functional impairment than BMI. ${ }^{9,10}$ Thus, gaining a better understanding of the effects of both general and abdominal obesity on functional outcomes is important for preserving independence in older adults.

Physical activity can help maintain and improve individuals' health by decreasing the risk of disability and delaying the onset of functional impairment. ${ }^{11,12} \mathrm{At}$ least 150 minutes of moderate physical activity and/or muscle strengthening activities per week is recommended for health maintenance, ${ }^{13}$ yet only $24 \%$ of adults aged 
65 years and more report engaging in any regular physical activity. ${ }^{14}$ Population estimates employing more objective measures of activity suggest even lower rates of engagement in older adults. ${ }^{15}$ Both physical inactivity and obesity are independently associated with functional decline and disability in older adults. However, less is known regarding the association between central adiposity, physical activity, and functional outcomes in older adults. ${ }^{16}$ Given the heightened risk of disability and potential loss of independence among older adults who are obese, there is an urgent need for effective lifestyle interventions that may aide in the prevention and management of obesity-related limitation and functional disability. The current study examined the impact of physical activity on physical and functional limitations among community-dwelling older adults with central adiposity.

\section{METHODS}

\section{Data Source}

This is a secondary analysis of existing data. Data were obtained from the 2006 and 2008 waves of the Health and Retirement Study (HRS), enhanced face-to-face interview. The HRS is a nationally representative panel survey of community-dwelling adults aged 50 years and more being conducted by the University of Michigan with support from the National Institute of Aging. The initial HRS sample was drawn in 1992 from a multistage, clustered area probability design of households, targeted individuals born between 1931 and 1941. Follow-up interviews and new cohort additions have occurred at regular intervals and have resulted in a nationally representative sample of American adults older than 50 years. A random one-half of the larger HRS sample was preselected to complete an enhanced face-to-face interview in 2006, which included physical and biomarker measurements. The second half of the HRS sample received the enhanced face-to-face interview in 2008. Inclusion of both 2006 and 2008 waves ensures that all HRS respondents who were eligible for and consented to the enhanced face-to-face interviews are included in the analyses. Additional descriptions of sampling procedures and study design are available online at: http://hrsonline.isr.umich.edu.

\section{Sample}

A total of 14724 eligible HRS respondents consented to physical assessments in 2006 and 2008. Of these, 10419 were aged 60 years and more. After eliminating respondents with missing waist circumference (WC), BMI, out of range values, and duplicate observations, there were 6279 subjects remaining for analyses. Subjects of all races/ethnicity were included in the sample. The study was exempt from Institutional Review Board approval at all respective institutions because of the deidentified nature of the data used.

\section{Measures}

\section{Adiposity}

Waist circumference was used as a proxy for abdominal adiposity. ${ }^{17}$ Waist circumference was assessed in person, using a nonelastic tape measure over a thin layer of clothing at the level of the respondents' navel while standing. The respondent was instructed to inhale and slowly exhale, holding their breath at the end of the exhale. Waist circumference was measured and recorded while holding the exhale. Individuals were categorized as having high abdominal adiposity in accordance with WC cut points established by the National Institutes of Health. ${ }^{18}$ Women with a WC of $88 \mathrm{~cm}$ or more and men with a WC of $102 \mathrm{~cm}$ or more were classified as having high abdominal adiposity; individuals with values below cut points were categorized as having low abdominal adiposity. Body mass index was also calculated where available for all respondents who consented to physical measures. In the HRS, weight was measured using a Healthometer $830 \mathrm{KL}$ (Medstock, Australia) scale and rounded to the nearest half pound. Weight for individuals more than $300 \mathrm{lb}$ was not recorded in the HRS as this was beyond the capacity of the measurement device. Height was measured by having the respondent stand barefoot with their heels and shoulders touching the wall. Individuals with a BMI between 18.5 and $24.9 \mathrm{~kg} / \mathrm{m}^{2}$ were classified as normal weight, 24.9 and $29.9 \mathrm{~kg} / \mathrm{m}^{2}$ as overweight, and $30 \mathrm{~kg} / \mathrm{m}^{2}$ or more as obese. ${ }^{18}$ Individuals who were underweight $(\mathrm{n}=61)$ were excluded from the analyses as this population is known to be at higher risk of frailty and disability. ${ }^{19}$

\section{Physical Activity}

Physical activity was assessed using a self-report physical health questionnaire as part of the HRS interview. Respondents were asked: "How often do you take part in sports or activities that are vigorous, such as running or jogging, swimming, cycling, aerobics or gym, workout, tennis, or digging with a spade or shovel: more than once a week, once a week, 1 to 3 times a month, or hardly ever or never?" and "How often do you take part in sports or activities that are moderately energetic, such as gardening, cleaning the car, walking at a moderate pace, dancing, floor, or stretching exercises: more than once a week, once a week, 1 to 3 times a month, or hardly ever or never?" For purposes of analyses, respondents reporting that they engaged in moderate or vigorous physical activity "once per week" or "more than once per week" were classified as active; those reporting activity less than once per week were classified as inactive ( $\mathrm{ref}=0$ ).

\section{Outcome Measures}

Physical limitations in the HRS was assessed using selfreport. Respondents were asked to report whether "they had difficulties (yes, no) in performing the following tasks 
because of a health or physical problem": walking several blocks, walking 1 block, sitting 2 hours, getting up from chair, climbing 1 flight of stairs, stooping, reaching arms, pulling/pushing large objects, lifting weights, and picking up a dime. All yes responses were compiled into a summary score in the HRS database ranging from 0 to 10 . Univariate analyses revealed that $67 \%$ of our sample reported at least 1 limitation. Thus, we chose a conservative cut point. For purposes of these analyses, respondents who reporting difficulty with performing 2 or more of the above tasks were classified as having physical limitations $(0=$ no limitation; $1=$ limitation).

\section{Functional limitations}

Activities of daily living (ADLs) and instrumental activities of daily living (IADLs) were used to assess functional limitations. ${ }^{20}$ Respondents were classified as having ADL limitations if they reported difficulty or inability with one or more of the following: bathing, dressing, eating, toileting, or getting out of bed. Respondents were classified as having IADL limitations if they reported difficulty with at least one of the following IADLs: preparing meals, managing money or needing help with house or yard work, using the phone, or taking medications All limitations reported in this article were measured in the HRS using self-report.
Respondents were instructed to exclude any difficulties that were expected to last less than 3 months. For all outcomes, responses were dichotomized $(0=$ no limitation; $1=$ limitation).

\section{Covariates}

Race and ethnicity (white, black, other) were entered as a categorical variable into the model. Age at the time of physical assessment, years of education completed, and the number of medical comorbidities were entered as continuous variables in the model. The comorbidity index ranged from 0 to 8 to reflect the number of diagnosed health conditions that the respondent self-reports. Conditions include diabetes, hypertension, lung disease, stroke, any cancer, arthritis, myocardial infarction, and chronic heart failure $\operatorname{coded}(0=$ no history; $1=$ positive history). Sex (male = 0 ; female $=1$ ) and current smoking status were entered as dichotomous variables into the model.

\section{Analyses}

The prevalence and odds of physical and functional limitations were examined within each WC category using chisquare and multivariate logistic regression. Unadjusted models were used to examine the impact of physical activity on functional limitations within each WC category

Table 1. Differences in Sample Characteristics by Waist Circumference Category

\begin{tabular}{|c|c|c|c|c|}
\hline Characteristic & $\begin{array}{c}\text { Total Sample } \\
\mathbf{N}=6279 \\
\text { Mean (SD) }\end{array}$ & $\begin{array}{l}\text { Low WC } \\
N=2353 \\
\text { Mean (SD) }\end{array}$ & $\begin{array}{l}\text { High WC } \\
\mathrm{N}=3926 \\
\text { Mean (SD) }\end{array}$ & $P$ Value $^{\mathrm{a}}$ \\
\hline Age, y & $68.9(6.09)$ & $69.2(6.17)$ & $68.7(6.03)$ & .0004 \\
\hline Education, y & $12.3(3.30)$ & $12.4(3.39)$ & $12.2(3.26)$ & .0022 \\
\hline Comorbidities & $2.7(1.22)$ & $2.6(1.23)$ & $2.8(1.21)$ & .0001 \\
\hline \multirow[t]{2}{*}{ Waist circumference, $\mathrm{cm}$} & $100.1(14.69)$ & $87.3(8.88)$ & $107.8(11.86)$ & .0001 \\
\hline & n (\%) & n (\%) & n (\%) & \\
\hline Sex & & & & $<.0001$ \\
\hline Male & $2875(45.8)$ & $1666(70.8)$ & $1209(30.8)$ & \\
\hline Female & $3404(54.2)$ & $687(29.2)$ & $2717(69.2)$ & \\
\hline Race $^{b}$ & & & & .05 \\
\hline White & 5106 (81.3) & $1947(82.7)$ & 3159 (80.5) & \\
\hline Black & 1015 (16.2) & $352(15.0)$ & 663 (16.9) & \\
\hline Other & $158(2.5)$ & $54(2.3)$ & $104(2.7)$ & \\
\hline Current smoker & $1517(24.2)$ & $615(26.1)$ & $902(23.0)$ & .006 \\
\hline Active & $3429(54.6)$ & $1392(59.2)$ & 2037 (51.9) & $<.0001$ \\
\hline Body mass index ${ }^{b}$ & & & & $<.0001$ \\
\hline Normal & $1523(24.5)$ & $1156(50.4)$ & $367(9.4)$ & \\
\hline Overweight & $2310(37.2)$ & $910(39.7)$ & $1400(35.7)$ & \\
\hline Obese & $2385(38.4)$ & $229(10.0)$ & $2156(55.0)$ & \\
\hline
\end{tabular}


as well as within each BMI category. Adjusted, multivariable models were adjusted for age, years of education, ethnicity, sex, smoking status, BMI, and the number of medical comorbidities. Waist circumference was used as a covariate in models stratified by BMI. All data were examined using SAS software version 9.3 (SAS Institute Cary, North Carolina) and weighted using the HRS respondentlevel weights for physical measures, which includes adjustments for sample selection probability and nonresponse. Detailed documentation regarding HRS sample weight calculations is available at http://hrsonline.isr.umich.edu/ sitedocs/wghtdoc.pdf. Statistical tests were 2-sided with $P$ values less than or equal to 0.05 . Confidence intervals excluding 1.0 were considered statistically significant.

\section{RESULTS}

Respondent demographics are presented in Table 1 by WC category. Over half of the sample was classified as having high abdominal adiposity (as measured by WC). Those in the high WC category were less educated, slightly younger, less active, and had a higher number of medical comorbidities than those in the low circumference category and were predominately female. Prevalence of physical and IADL limitations was notably higher among those with high WC than among those with low WC, but there were no significant differences in the prevalence of ADL limitation between low- and high-WC groups. Prevalence of physical, ADL, and IADL limitations is reported in Table 2 by WC. We also report prevalence by BMI for purposes of comparison.

Odds ratios and confidence intervals for functional limitations among physically active older adults as compared with older adults who report low or no activity are presented in Table 3. A total of 1503 of the sample had nonpositive weights and were automatically excluded from the analyses (they did not contribute statistically to the analyses). Both unweighted $(\mathrm{n}=6279)$ and weighted $(\mathrm{n}=$

Table 2. Prevalence of Physical and Functional Limitations in Physically Active, Community-Dwelling Older Adults by Waist Circumference and Body Mass Index

\begin{tabular}{|c|c|c|c|}
\hline \multirow[b]{2}{*}{ Obesity Category } & Physical Limitations & $A D L$ & IADL \\
\hline & $n(\%)^{a}$ & $n(\%)^{a}$ & $n(\%)^{a}$ \\
\hline \multicolumn{4}{|c|}{ Waist circumference } \\
\hline Low & $11091(46.4)$ & 595 (49.8) & $1011(43.9)$ \\
\hline High & $2163(55.1)$ & $1144(50.0)$ & $1823(47.3)$ \\
\hline \multicolumn{4}{|l|}{ Body mass index } \\
\hline Normal & $721(47.3)$ & $417(53.1)$ & $702(46.8)$ \\
\hline Overweight & 1137 (49.2) & $592(47.0)$ & $997(44.1)$ \\
\hline Obese & 1356 (56.9) & $710(50.5)$ & $1100(47.0)$ \\
\hline
\end{tabular}

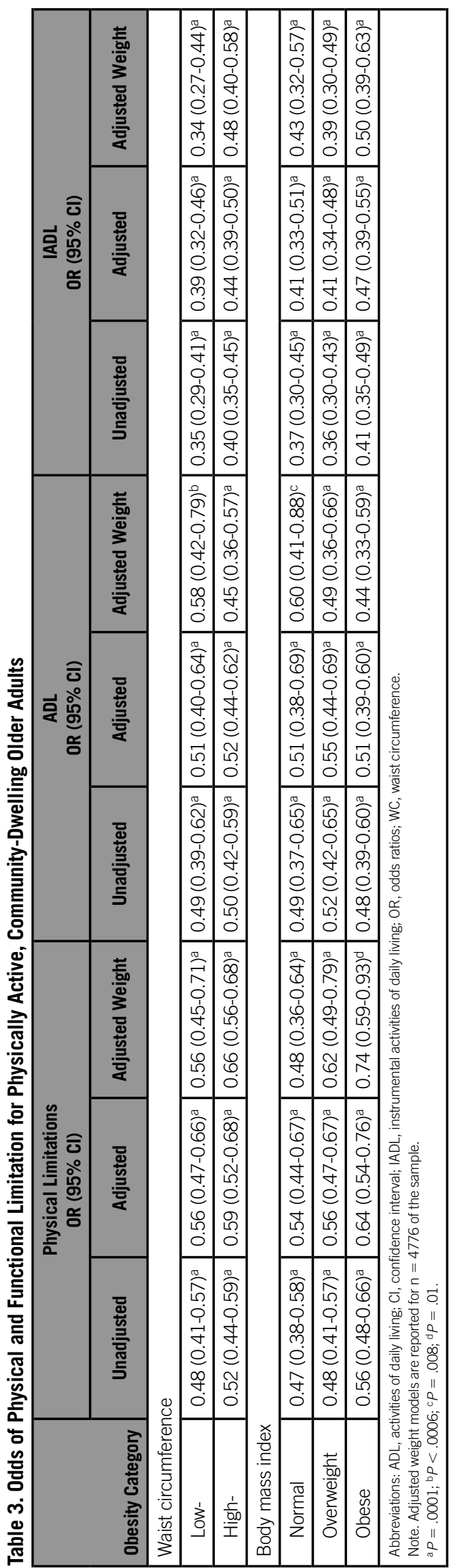

Volume $00 \bullet$ Number $00 \bullet x x x x 2015$ 
4776) adjusted models are reported in Table 3. However, a comparison of weighted and unweighted models revealed no meaningful differences in the results. Results discussed in text are unweighted. In the high-WC group, physical activity was associated with significantly lower odds of physical, ADL, and IADL limitations. The association remained after adjusting for age, sex, race/ethnicity, education, smoking status, BMI, and the number of comorbidities. Physical activity was also associated with lower odds of physical and functional limitation among those with low WC. Consistent with existing literature, ${ }^{2}$ having a higher number of comorbidities was associated with higher odds of limitation in all functional domains among older adults with high and low WC. Similar results were obtained using BMI categories (Table 4). Sex and ethnicity were also found to be differentially associated with odds of physical and functional impairment among older adults with low WC. Nonwhite status was marginally associated with lower odds of physical limitation (odds ratio, 0.82 [0.67-01.00]), but not ADL or IADL limitation; being female was associated with higher odds of ADL limitation (odds ratio, 1.52 [1.16-1.99]).

\section{DISCUSSION}

The current study examined the impact of physical activity on prevalence and odds of physical and functional limitations in obese older adults. Obesity was categorized using both WC and BMI criteria. Our results indicate that regardless of the obesity criteria used (WC or $\mathrm{BMI}$ ), physical activity is significantly associated with lower odds of physical and functional limitations in older adults who are obese. Obese older adults in the current sample who reported engaging in moderate/vigorous physical activity at least once per week were significantly less likely to report physical and functional limitations than those who reported low/no physical activity. The finding suggests that the benefits of physical activity are not limited to normalweight older adults, but may moderate the negative impact of carrying excess weight on physical and functional limitations among those who are obese. Our results are also encouraging in that the deleterious effects of increasing comorbidities did not negate the positive impact of physical activity on physical and functional limitations in the current study. This is a particularly important consideration among obese elders who are more likely to present with multiple health conditions and suggests that even minimal engagement in moderate/vigorous physical activity is associated with reduced odds of impairment in spite of multiple health conditions.

Our findings also suggest that there are important sex and ethnic differences in the association between weight and physical and functional outcomes. Consistent with observations from studies of obese older persons and recently published trends, ${ }^{4}$ there was a higher proportion of women classified as obese than men. This was particularly true when using WC guidelines and confirms recent reports that abdominal weight gain among women is occurring at a much greater rate than among men. ${ }^{7}$ However, normal and overweight women in this sample tended to be at higher odds of ADL limitation than men, but the odds of ADL limitation did not differ between men and women among the obese as might be expected. This may in part be due to biological differences in body composition between men and women. ${ }^{21}$ We also found that nonwhite status was associated with lower odds of physical limitations in higher weight categories in spite of reports consistently placing minorities at higher ends of the weight spectrum. These findings suggests that the relationship between excess weight and functional outcomes in both minorities/nonwhites and older women is complex and merits further investigation. ${ }^{22}$ Current research is underway by our group to further examine the nature of these ethnic and sex differences.

Primary strengths of the current study include analyses of data based in a large, well-characterized, nationally representative community-dwelling sample, and the availability of 2 objective measures of adiposity. Although the current results are based on a nationally representative

Table 4. Independent Impact of Increasing Comorbidities on Physical and Functional Limitations in Physically Active, CommunityDwelling Older Adults

\begin{tabular}{|c|c|c|c|c|c|c|}
\hline Obesity Category & \multicolumn{2}{|c|}{$\begin{array}{l}\text { Physical Limitations } \\
\text { OR }(95 \% \mathrm{CI})\end{array}$} & \multicolumn{2}{|c|}{$\begin{array}{c}\text { ADL } \\
\text { OR }(95 \% \mathrm{CI})\end{array}$} & \multicolumn{2}{|c|}{$\begin{array}{c}\text { IADL } \\
\text { OR (95\% Cl) }\end{array}$} \\
\hline \multicolumn{7}{|c|}{ Waist circumference } \\
\hline Low & $1.48(1.37-1.59)^{a}$ & $1.46(1.32-1.61)^{a}$ & $1.13(1.02-1.25)^{a}$ & $1.21(1.05-1.39)^{a}$ & $1.54(1.43-1.67)^{\mathrm{a}}$ & $1.67(1.50-1.86)^{\mathrm{a}}$ \\
\hline High & $1.48(1.40-1.6)^{a}$ & $1.54(1.42-1.67)^{a}$ & $1.23(1.15-1.33)^{a}$ & $1.21(1.09-1.34)^{a}$ & $1.00(0.85-1.17)^{\mathrm{a}}$ & $1.65(1.52-1.79)^{a}$ \\
\hline \multicolumn{7}{|l|}{ Body mass index } \\
\hline Normal & $1.45(1.32-1.58)^{a}$ & $1.41(1.25-1.58)^{a}$ & 1.09 ( 0.96-1.24) & 1.09 ( 0.92-1.29) & $1.55(1.41-1.71)^{\mathrm{a}}$ & $1.73(1.52-1.97)^{\mathrm{a}}$ \\
\hline Overweight & $1.50(1.39-1.62)^{a}$ & $1.54(1.38-1.71)^{a}$ & $1.21(1.10-1.34)^{\mathrm{a}}$ & $1.22(1.07-1.38)^{\mathrm{b}}$ & $1.52(1.41-1.65)^{a}$ & $1.56(1.40-1.73)^{\mathrm{a}}$ \\
\hline Obese & $1.45(1.34-1.57)^{a}$ & $1.53(1.37-1.70)^{a}$ & $1.25(1.14-1.38)^{a}$ & $1.28(1.11-1.46)^{\mathrm{c}}$ & $1.63(1.50-1.77)^{a}$ & $1.72(1.55-1.91)^{\mathrm{a}}$ \\
\hline
\end{tabular}


population, the results are limited in generalizability in that they are cross-sectional in nature. Examination of longitudinal data is needed to understand the differential impact of changes in physical activity and changes in weight on physical and functional limitations over time, particularly in women and minorities. Additional limitations include that there were no objective measures of physical activity used in the survey and the simple count measure of comorbidity used for analyses does not take disease severity into account. ${ }^{23}$ Imprecise measurements of physical activity may have affected study results including prevalence estimates reported here.

\section{CONCLUSIONS}

Engagement in moderate/vigorous intensity exercise at least once per week is associated with lower odds of physical and functional impairment in obese older adults. Encouraging physical activity in normal weight and obese older adults may help to lower the odds of physical and functional impairment. Additional research is needed to examine the long-term benefits of physical activity in older adults who remain obese.

\section{ACKNOWLEDGEMENTS}

We acknowledge James A. Blumenthal, Gerda Fillenbaum, and Celia Hybels for their reviews of previous versions this article.

\section{REFERENCES}

1. Gregg EW, Cheng YJ, Cadwell BL, et al. Secular trends in cardiovascular disease risk factors according to body mass index in US adults. JAMA. 2005;293(15):1868-1874.

2. Penn DM, Fischer JG, Sun Lee J, Hausman DB, Johnson MA. High BMI and waist circumference are associated with a high prevalence of comorbidities in older Americans act programs in Georgia senior centers. J Nutr Health Aging. 2009;13(9):827-832.

3. Chen H, Guo X. Obesity and functional disability in elderly Americans. J Am Geriatr Soc. 2008;56(4):689-694.

4. Flegal KM, Carroll MD, Kit BK, Ogden CL. Prevalence of obesity and trends in the distribution of body mass index among US adults, 1999-2010. JAMA. 2012;307(5):491-497.
5. Ogden CL, Carroll MD, Kit BK, Flegal KM. Prevalence of obesity in the united states, 2009-2010. NCHS Data Brief. 2012;82:1-8.

6. Wang Y, Beydoun MA. The obesity epidemic in the United Statesgender, age, socioeconomic, racial/ethnic, and geographic characteristics: a systematic review and meta-regression analysis. Epidemiol Rev. 2007:29(1):6-28.

7. Li C, Ford ES, McGuire LC, Mokdad AH. Increasing trends in waist circumference and abdominal obesity among US adults. Obesity (Silver Spring). 2007; 15(1):216-224

8. Houston D, Stevens J, Cai J. Abdominal fat distribution and functional limitations and disability in a biracial cohort: the Atherosclerosis Risk in Communities Study. Int J Obes. 2005;29(12):1457-1463.

9. Janssen I, Katzmarzyk PT, Ross R. Waist circumference and not body mass index explains obesity-related health risk. Am J Clin Nutr. 2004;79(3): 379-384.

10. Angleman S, Harris T, Melzer D. The role of waist circumference in predicting disability in periretirement age adults. Int J Obes. 2005;30(2) 364-373.

11. Paterson DH, Warburton DE. Physical activity and functional limitations in older adults: A systematic review related to Canada's physical activity guidelines. Int J Behav Nutr Phys Act. 2010;7:38.

12. Riebe D, Blissmer BJ, Greaney ML, Garber CE, Lees FD, Clark PG. The relationship between obesity, physical activity, and physical function in older adults. J Aging Health. 2009;21(8):1159-1178.

13. US Department of Health and Human Services. Physical activity guidelines for Americans. http://www.health.gov/paguidelines. Published 2008. Accessed March 1, 2014.

14. Sun F, Norman IJ, While AE. Physical activity in older people: a systematic review. BMC Public Health. 2013;13:449.

15. Tucker JM, Welk GJ, Beyler NK. Physical activity in U.S.: Adults compliance with the physical activity guidelines for Americans. Am J Prev Med. 2011;40(4):454-461.

16. Koster A, Penninx BW, Newman AB, et al. Lifestyle factors and incident mobility limitation in obese and non obese older adults. Obesity. 2007;15(12):3122-3132.

17. Pouliot M, Després J, Lemieux S, et al. Waist circumference and abdominal sagittal diameter: best simple anthropometric indexes of abdominal visceral adipose tissue accumulation and related cardiovascular risk in men and women. Am J Cardiol. 1994;73(7):460-468.

18. US Department of Health and Human Services. Clinical Guidelines on the Identification, Evaluation, and Treatment of Overweight and Obesity in Adults. Washington, DC: Public Health Service, National Institutes of Health, National Heart, Lung, and Blood Institute; 1998. http://www.nhlbi.nih.gov/ guidelines/obesity/ob_gdlns.pdf. Accessed March 1, 2014.

19. Bowen ME. The relationship between body weight, frailty, and the disablement process. J Gerontol B Psychol Sci Soc Sci. 2012;67(5):618-626.

20. Saliba D, Orlando M, Wenger NS, Hays RD, Rubenstein LZ. Identifying a short functional disability screen for older persons. J Gerontol A Biol Sci Med Sci. 2000:55(12):M750-M756.

21. Goodman-Gruen D, Barrett-Connor E. Sex differences in measures of body fat and body fat distribution in the elderly. Am J Epidemiol. 1996;143(9):898-906.

22. Carroll JF, Chiapa AL, Rodriquez M, et al. Visceral fat, waist circumference, and BMI: impact of race/ethnicity. Obesity. 2008;16(3):600-607.

23. Perkins AJ, Kroenke $\mathrm{K}$, Unützer J, et al. Common comorbidity scales were similar in their ability to predict health care costs and mortality. J Clin Epidemiol. 2004;57(10):1040-1048. 